\title{
DESIGN AND CHARACTERIZATION OF A DESK-TOP LASER PLASMA X-RAY SOURCE FOR RADIOBIOLOGY STUDIES ${ }^{*}$
}

\author{
Daniel Adjei ${ }^{*{ }_{1}, 2,4}$, Anna Wiechec3,Przemyslaw Wachulak ${ }^{1}$, MesfinGetachew Ayele', \\ Janusz Lekki3, Wojciech M. Kwiatek3, Andrzej Bartnik', \\ Ladislav Pina ${ }^{4}$, Henryk Fiedorowicz ${ }^{1}$
}

\author{
${ }^{1}$ Institute of Optoelectronics, Military University of Technology, Warsaw, Poland \\ ${ }^{2}$ Radiation Protection Institute, Ghana Atomic Energy Commission, Legon, Accra, Ghana \\ 3Institute of Nuclear Physics, Polish Academy of Sciences, Cracow, Poland \\ 4Faculty of Nuclear Sciences and Engineering Physics, Czech Technical University in Prague, Prague, Czech Republic
}

\begin{abstract}
A compact desk-top laser-produced plasma source of soft X-rays for radiobiology research is presented. The source is based on a double-stream gas puff target and delivers nanosecond pulses of soft X-rays in the "water window" spectral range at a fluence of about $4.23 \times 10^{3}$ photon $/ \mu m^{2}$ per pulse on a sample placed inside the vacuum source chamber and about $2.60 \times 1 O^{2}$ photon $/ \mu m^{2}$ per pulse on a wet sample located outside the chamber in the Heenvironment. The source has been used to irradiate pBR322 plasmid DNA both in vacuum and the He-environment conditions. Single and double strand breaks were quantified by gel electrophoresis. The number of strand breaks increased with the increasing dose of the "water window" soft X-rays. The strand breaks of plasmid solution irradiated in helium condition may be associated with damage from water-free radicals.
\end{abstract}

Key words: Laser-produced plasma, soft X-rays, radiobiology, gas puff target, water window

DOI: $10.21175 /$ RadJ.2016.02.016

\section{INTRODUCTION}

Application of soft X-rays $(<12 \mathrm{keV})$ in radiobiology has been demonstrated in various experiments [1-17]. A number of reports have reviewed that the biological damage, produced by this radiation, was found very often more effective as compared with hard X-rays $[6,7,14]$. This can be explained by the high absorption and relatively low penetration (as compared with hard X-rays) of the radiation in cells and cellular components, allowing high proportion of the energy to be deposited per unit mass of the sample. This particularly applies to soft X-rays in the unique spectral region between the oxygen K-edge at $2.3 \mathrm{~nm}$ and the carbon k-edge at $4.4 \mathrm{~nm}$, called "water window", where the carboncontaining proteins show much larger absorption than the oxygen-containing water, thus allowing a high number of photons to be delivered to the sample. This makes the use of soft X-rays in the "water window" spectral region a useful method for investigating the mechanisms of radiation damage at high-dose loads. Radiations in these experiments were generated with X-ray tubes and synchrotrons, however, the latter sources have limited access.

Recently, application of laser plasma soft X-ray sources in radiobiology has attracted much interest, because of the possibility irradiation of a sample at high-dose loads and rates. Additionally, very short pulses emitted from these sources may be useful to understand the mechanism underlying the biological response to radiation. Laser plasma soft
$\mathrm{X}$-rays sources for radiobiology experiments were proposed more than 20 years ago [18-19], however, such sources were based on the use of a laser interacting with a solid target. Laser plasma sources based on solid targets produce debris which may cause degradation to the optical elements of the system and the sample under irradiation. Also, solid target laser plasma sources emit X-rays with high energy spread and the reproducibility of the radiation dose might be of a challenge [20].

The debris problem can be solved by the use of a double-stream gas puff target approach that was proposed at the Institute of Optoelectronics, Military University of Technology [21]. Efficient soft X-ray emission in the "water window" spectral region has been demonstrated from laser plasma produced using the gas puff target [22]. The laser plasma soft X-ray source based on the xenon gas puff target irradiated with laser pulses at the PALS laser facility has been successfully used in the radiobiology experiments [23]. However, because of the limited access to this large scale laser facility, there is a strong need to develop a laboratory setup based on a compact laser plasma X-ray source to be used by many users for potential radiobiology experiments.

Soft X-rays photons are easily attenuated to less than $10 \%$ at transmission path length of $\sim 1 \mathrm{~cm}$ of air [24]. Thus, there are useful in high vacuum condition. In order to effectively use such photons for irradiating live or wet samples will require that the X-ray attenuation is prevented as much as

\footnotetext{
* The paper was presented at the Third International Conference on Radiation and Applications in Various Fields of Research (RAD 2015), Budva, Montenegro, 2015.
}

**daniel.adjei@wat.edu.pl 
D. Adjei et al., Design and Characterization of a Desk-Top Laser ..., Rad. Applic., 2016, 1, 2, 88-94

possible. One of the possible ways is to transmit the $\mathrm{X}$-ray beam through a plume of helium which allows about $99 \%$ transmission of such photons.

An application of a "stand alone" compact soft $\mathrm{X}$-ray source, developed and optimised for high emission in the X-ray "water window" spectral range, for radiobiology studies is presented in this study. The source has been developed based on our existing laser plasma source of the „water window” $\mathrm{X}$-rays for application in microscopy [22].

\section{MATERIALS AND METHODS}

\subsection{Design and development of a nanosecond} laser plasma $X$-ray source

A compact laser plasma system with a unique sample irradiation mounting systems has been developed for radiobiology experiments. Aspects of the source development are described in our previous paper [25]. The source is based on focusing a laser (Nd:YAG laser, $1.06 \mu \mathrm{m}$ wavelength, $\sim 740 \mathrm{~mJ}$ maximum energy and $4 \mathrm{~ns}$ pulse duration) beam onto a double stream gas puff target in a vacuum-chamber. Here, we have used $\mathrm{Ar} / \mathrm{He}$ gas puff target, which combined with different spectral absorbers give maximum emission in the X-ray "water window" spectral wavelength range.

The source has been additionally equipped with new systems to mount samples to be able to irradiate biological samples both in vacuum and in He-environment. To irradiate samples in vacuum, the sample mounting system was made in a form of a circular disc with $8 \mathrm{~mm}$ diameter holes arranged in $360^{\circ}$ and mounted on a motorized rotation stage. The layout allowed eight samples to be irradiated in vacuum in order to limit the time spent on pumping and venting the chamber to change the samples. During irradiation of a particular sample, the remaining samples are shielded from the radiation beam by a $200 \mu \mathrm{m}$ thick of aluminum foil glued to a $1 \mathrm{~mm}$ thick of plastic. Precise positioning of the sample in the X-ray beam was executed by remotely moving the sample into the beam with the stage controller. The sample placed in the vacuum chamber could be visualized through a glass window mounted on top of the chamber.

A vacuum of $\sim 10^{-2}$ mbar during operation was achieved with the use of a turbo molecular pump backed by a rotary pump. Though the vacuum condition is relatively high, the transmission of argon for a few cm path lengths is high [24].Thin film spectral filters were required to select specifically the X-ray "water window" spectral range. The filters were accommodated in the chamber with a special home-made holder which allowed uniform pressure distribution around the filters. Thus, it was possible to avoid any damage which might be due to different pressure gradient on the filters.
To allow irradiation of wet biological samples, a second sample mounting system was mounted outside, on top of the vacuum chamber, and correctly aligned to allow the soft X-rays to emerge through a $2 \times 2 \mathrm{~mm}^{2}$ silicon nitride window, $200 \mathrm{~nm}$ thick (Silson Ltd, UK). Another unique feature of the source is that samples are mounted in a vertical position. A plastic sealed container, flushed with He-gas, was mounted directly above the window to avoid the strong absorption of the soft X-ray beam by air in the atmospheric condition. The container is separated by an O-ring to provide a proper sealing and prevent any damage to the window. The experimental arrangement for irradiating samples outside the vacuum chamber is schematically shown in Fig.1.

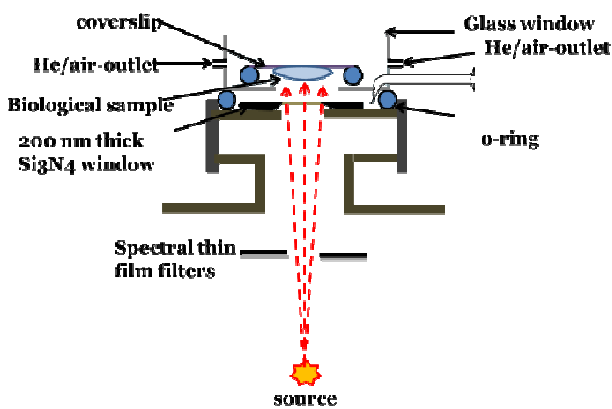

Figure 1. Schematic view of the experimental arrangement for irradiating biological samples in helium environment

\subsection{Source characterization measurements}

The use of the gas puff target required proper optimization of the source parameters. Here, the backing pressures of the argon and helium gases were optimized to achieve higher photon flux. Similarly, the time delays of the opening and closing of the valve which was synchronized with the laser power supply was optimized. The basic concept of the source optimization is described elsewhere[22, 25].The position of the electromagnetic valve was optimized to align it so that the nozzle is about $1.5 \mathrm{~mm}$ away from the focus of the laser beam, and located in the center of the chamber whiles providing optimum gas density for higher photon emission.

The photon fluence measurements have been performed for both $\mathrm{He}$ environment and vacuum conditions using calibratedAXUV-10osilicon PIN photodiode (International Radiation Detectors Inc., USA) with a $10 \times 10 \mathrm{~mm}^{2}$ active area. During the photon fluence measurements in vacuum, the soft $\mathrm{X}$-rays were transmitted through the silicon nitride and $\mathrm{Ti}$ filters located $30 \mathrm{~mm}$ and $36 \mathrm{~mm}$, respectively, upstream of the source. The $30 \mathrm{~mm}$ distance between the interaction point and the silicon filter was sufficient to prevent damaging the filter by the plasma shockwave and the pressure from the gas puff target. The efficiency of the detector depends on the X-ray energy; hence, the 
D. Adjei et al., Design and Characterization of a Desk-Top Laser ..., Rad.Applic., 2016, 1, 2, 88-94

quantum efficiencies (provided by the manufacturer) were normalized according the obtained spectra. This was achieved by taking into account the normalized transmission coefficients of each spectral line by the corresponding wavelength dependent quantum efficiency. The detector was operated with reverse bias voltage of $\sim 28 \mathrm{~V}$ and coupled to a $4 \mathrm{GHz}$ bandwidth Tektronix DPO 70404 Digital Phosphor oscilloscope (DPX ${ }^{\circledR}$ Technology, USA) with impedance of $50 \mathrm{ohm}$. The time integrated voltage was converted to the stored charge, the charge then (in number of electrons) was converted to number of photons by the conversion factor given for a specific wavelength. During the measurements for the $\mathrm{He}$ environment a silicon nitride foil of $200 \mathrm{~nm}$ thick was used as a window separating the vacuum and the He-environments. The detector was placed in the same position as the sample would be placed in the He-environment.

Further investigation has been carried out to check the contribution of ultraviolet (UV) radiation transmitted through the $\mathrm{Si}_{3} \mathrm{~N}_{4}$ to the sample in the He-environment. This part of the experiment has been performed by introducing $2 \mathrm{~mm}$ thick calcium fluoride $\left(\mathrm{CaF}_{2}\right)$ and $1.5 \mathrm{~mm}$ thick glass absorbers. The $\mathrm{CaF}_{2}$ filter which has excellent transmission from $130 \mathrm{~nm}$ to $10 \mu \mathrm{m}$ wavelength made possible to check the contribution of UV and visible light (VIS).Pinhole images of the source were taken and a comparison of the relative intensities of the source when with and or without $\mathrm{CaF}_{2}$ or glass filters was introduced.

\subsection{Sample preparation for irradiation}

Radiobiology experiment was carried to demonstrate the applicability of the source. In this experiment, stock pBR322 (4361 bp) plasmid solution (Thermo Fisher Scientific) was prepared for irradiation. Volumes of $1.2 \mu \mathrm{l}$ corresponding to 600 ng of the plasmid DNA were deposited onto glass cover slips. For the vacuum irradiation condition, the samples were left to dry in a nitrogen atmosphere for 30 minutes. Then the cover slip (with the sample surface facing the source) was placed in the source chamber. The schematic view of the experimental procedure employed in irradiating samples in vacuum is as shown in Fig. 2.

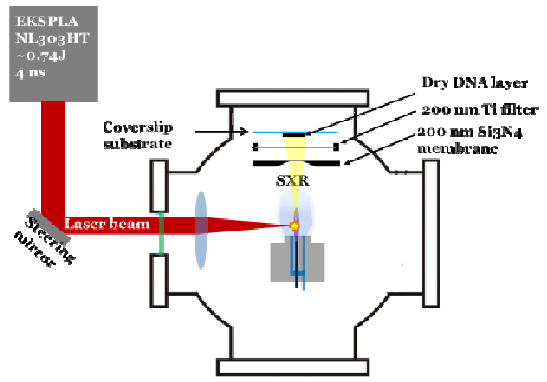

Figure 2.Schematic view of the experimental setup for irradiating biological samples in the source chamber in vacuum
It was possible to irradiate 8 samples with one of the samples not in the vicinity of the radiation as a controlled sample. During irradiation, each sample was irradiated differently according to the number of specified soft X-ray pulses whiles the remaining samples were shielded from the radiation by $\mathrm{Al}$ and plastic foil absorbers. Samples are irradiated with10, 20, 30, 40, 50, and 60X-ray pulses. Each pulse corresponds to $\sim 297 \mathrm{~Gy}$. The dosimetry was performed following the procedure reported by Yokoya et al (1999) [26] by taking into consideration the stoichiometric confidents of the pBR322 plasmid DNA. Hence, the mass absorption coefficient of the sample was obtained using Henke's data [24].

Similarly, $1.2 \mu \mathrm{l}$ volumes of the plasmid DNA solution were pipetted onto glass cover slips (but not subjected to the drying condition) and irradiated in the He-environment, outside the vacuum chamber. The samples were irradiated with10, 100, $200,500,1000,1500$, and $2000 \mathrm{X}$-ray pulses, with each pulse corresponding to $\sim 18 \mathrm{~Gy}$.

\subsection{DNA damage analysis by gel electrophoresis}

Each dried irradiated DNA sample was immediately dissolved in $10 \mu \mathrm{l}$ of distilled water. Volume of $5 \mu \mathrm{l}$ of the solution was mixed with $1 \mu \mathrm{l} 6 \mathrm{X}$ DNA Loading Dye (bromophenol blue: 30\% (w/v), glycerol/o.25\% (w/v), xylene cyanol/o.25\% (w/v)), (Thermo Fisher Scientific). The DNA fragments, after irradiation, were separated in a 1\% agarose gel in $0.5 \mathrm{X}$ Tris-acetate-EDTA (TAE) buffer $(20 \mathrm{mM}$ Tris, $10 \mathrm{mM}$ solution acetate, $1 \mathrm{mM}$ Ethylenediaminetetraacetic acid, EDTA, $P H=8.0$ ) and ran at $60 \mathrm{~V}\left(6 \mathrm{Vcm}^{-1}\right)$ of $20 \mathrm{~mA}$ current for 2 hours. The DNA samples and the agarose gels after electrophoresis were stained in ethidium bromide, $(1 \% \mathrm{w} / \mathrm{v})$, for 3 hours and rinsed in distilled water for 30 minutes.

Images of the gel were taken on a $312 \mathrm{~nm}$ UV transilluminator (Polgen Company, Poland) coupled to a digital camera interfaced onto a HP ProBook computer with TotalLab Quant Software used to acquire the gel images for analysis. The obtained images were imported to ImageJ software to quantify the relative intensity of each band. The amounts of supercoiled (S), circular (C) and linear (L) plasmid DNA were determined from the integrals of their respective peaks recorded in the ImageJ software according to the relation:

$$
S=\frac{s}{s+c+l}
$$

where $s, c$ and $l$ are the resulted peak areas of the super coiled, open circular and linear forms, respectively [27]. Following a similar procedure, the yields of the open circular $-\mathrm{C}$, and linear $-\mathrm{L}$, forms were estimated. 
D. Adjei et al., Design and Characterization of a Desk-Top Laser ..., Rad. Applic., 2016, 1, 2, 88-94

\section{EXPERIMENTAL RESULTS AND DISCUSSION}

\subsection{Source characterization and optimization}

Proper optimization of the source is always a priority to ensure maximum photon fluence on a sample. It was done by determination of the time delays between the trigger pulses opening of the valves and the synchronization pulse of the laser for which the maximum soft X-ray emission was observed. The maximum emission was obtained when the time delays for the inner and outer valves were $0.75 \mathrm{~ms}$ and $0.4 \mathrm{~ms}$, respectively. The optimum backing pressures of the argon and helium were kept at 10 and 6 bars, respectively, to give the maximum emission.

For the photon flux measurements, the number of photons transmitted through the silicon nitride and the titanium filters to the detector plane per single pulse was estimated to be $(3.48 \pm 0.35) \times 10^{8}$ photon $/ \mathrm{cm}^{2}$. Thus, $(5.44 \pm 0.55) \times 10^{12}$ photon $/ \mathrm{sr}$ could be calculated in the detector plane. The conversion efficiency of laser energy into energy of soft X-rays was $\sim 0.57 \%$.

The number of soft X-ray photons to be delivered to a biological sample placed inside the vacuum chamber at the sample plane $(36 \mathrm{~mm}$ upstream of the source), behind $\mathrm{Ti}$ and $\mathrm{Si}_{3} \mathrm{~N}_{4}$ filters, is approximately $4.20 \times 10^{3}$ photon $/ \mu \mathrm{m}^{2}$ per pulse. The photon flux delivered by our source is comparable with those achieved in synchrotron microbeam system [25], though it is the state of art system, for radiobiology studies.

For the He-environment irradiation condition, we have chosen $2 \times 2 \mathrm{~mm}^{2}$ size of $\mathrm{Si}_{3} \mathrm{~N}_{4}$ window to let the X-rays out of the vacuum chamber to the sample. The relatively small size was chosen to allow the window to stand the vacuum-atmospheric pressures. The distance between the sample and the exit window allowed the spread of the X-ray photons to cover the entire sample. The number of soft X-ray photons to be delivered to a sample in the Heatmosphere was $2.6 \times 10^{2}$ photons $/ \mu \mathrm{m}^{2}$ per pulse. For the helium condition irradiation, the photons are transmitted through the $200 \mathrm{~nm} \mathrm{Si}_{3} \mathrm{~N}_{4}$ and a plume of helium of path length of $\sim 1 \mathrm{~cm}$. We have used the $\mathrm{Si}_{3} \mathrm{~N}_{4}$ window which is capable to absorb the extreme ultraviolet (EUV) and UV rays while allowing excellent transmission of the "water window" X-rays. This was confirmed by the investigation to check the contribution of UV transmitted through the $200 \mathrm{~nm} \mathrm{Si}_{3} \mathrm{~N}_{4}$ and the plume of helium. Fig. 3shows the pinhole camera images of the source with or without glass or $\mathrm{CaF}_{2}$ absorbers. It can be seen that the image of the source through the $\mathrm{Si}_{3} \mathrm{~N}_{4}$ window was much brighter than was with glass or CaF2 absorbers. However, there was no difference between the images with glass and $\mathrm{CaF}_{2}$. It can be concluded that there was insignificant contribution of UV for irradiating sample in the He atmosphere. It is likely that the UV is absorbed by the $\mathrm{Si}_{3} \mathrm{~N}_{4}$ window.

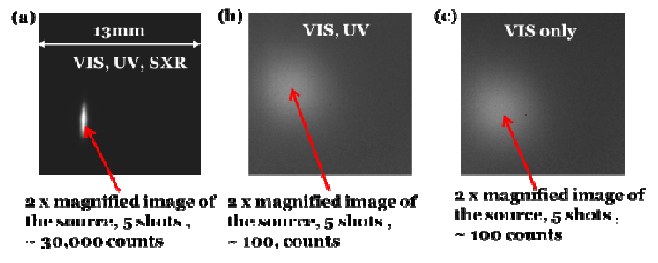

Figure 3.The image of the source obtained through a 200 $\mathrm{nm} \mathrm{Si} \mathrm{N}_{3}$ membrane: (a) withoutCaF $\mathrm{F}_{2}$ and glass absorbers,

(b) filtered by $\mathrm{CaF}_{2}$ only, (c) filtered by glass

\subsection{Radiobiological experiment}

The pBR322 plasmid DNA (4361 bp) has been chosen as a model for an initial soft X-ray radiobiological experiment to demonstrate the applicability of the source. When irradiated, the plasmid DNA changes its form fromthe native supercoiled (S) to the relaxed (R) form indicating production of single strand breaks (SSB). When one double strand break (DSB), or two single strand breaks within certain distance on the opposite strands of the double helix are induced by soft X-ray radiation (i.e., direct action) and/or reactive species generated by the radiation in water (i.e., indirect action) the plasmid changes its conformation to the linear form (L).

The dried plasmid DNA sample (with the buffer solute) covered an area of $\sim 2 \times 2 \mathrm{~mm}^{2}$ on the cover slip. The thickness of the dried plasmid DNA sample on the cover slip was estimated by atomic force microcopy measurements to be $\sim 1 \mu \mathrm{m}$ thick. More than $85 \%$ of the plasmid DNA used was characterized to be in the supercoiled form. A series of DNA samples were exposed in vacuum using the experimental layout reported here at increasing soft $\mathrm{X}$-ray pulses. The dried DNA irradiated in vacuum showed remarkable exponential decrease in the supercoiled form of the DNA with corresponding exponential increase in the linear and relaxed forms as a function of photon flux. The control samples remained almost the same quantity of supercoiled form when placed in vacuum with the irradiated samples. Thus, the vacuum condition did not affect the dried supercoiled form of the DNA. This shows the capability of the "water window" X-ray photons in inducing strand breaks in plasmid DNA. To further understand the efficiency of the source, the obtained data was fitted to a model proposed by McMahon and Currel [28] as shown in Fig. 4. 


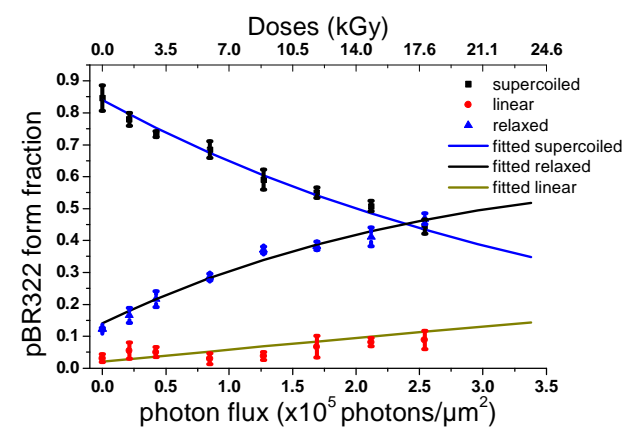

Figure 4.Fraction of yields of different forms of pBR322 plasmid DNA irradiated in vacuum as a function of photon, the lines indicate the fitting model proposed by McMahon and Currel[28]. Vertical error bars indicate the standard error of the mean. Horizontal errors are not included on the graph but correspond to about $10 \%$ random uncertainty on the photon flux measurements on the photodiode and fluctuation in the density of the gases

It shows that the exponential decrease in supercoiled form with the corresponding increase in linear and relaxed forms follow the established trend of plasmid DNA damage after irradiation. From the model fitting, the yields of the SSB and DSB were estimated to be $6.03 \times 10^{-3} \mathrm{SSB} / 1 \mathrm{Mbp} / \mathrm{Gy}$ and $9.79 \times 10^{-4} \mathrm{DSB} / 1 \mathrm{Mbp} / \mathrm{Gy}$, respectively. The single strand breaks cross-section, бSSB was estimated to be $2.33 \times 10^{-18} \mathrm{~m}^{2}$ and that of the DSB, $\sigma_{\mathrm{DSB}}$, was $4.27 \times 1 \mathrm{O}^{-19} \mathrm{~m}^{2}$. The measured cross-section for the SSB induction in plasmids irradiated was slightly lower than the cross-sections measured with monochromatized synchrotron radiation [29].

This may be explained by the difference in irradiation conditions, such as DNA concentration and the specific energies of photons delivered to the samples. From the cross-sections, it is expected that the photons are predominately absorbed by the Kshell electrons of the carbon, nitrogen and oxygen atoms in the DNA. Similarly, taking the ratio of the SSB to DSB gives an approximate value of 7 which is less than that observed by Yokoya et al (1999) [26]. Probably, in part, due to the plasmid DNA concentration but comparable with that observed by Eschenbrenner et al.[26], which varied between 4.4 and6.4 when pBs and pSP plasmids were irradiated to 388,435 and $573 \mathrm{eV}$ soft X-rays from synchrotron source. The results show that the source, though in its initial stage of development, could be useful for prospective radiobiology experiments and could be added to the few laser plasma X-ray sources dedicated for radiobiology experiments. Similarly, the fraction of the different forms of the pBR322 plasmid DNA in TAE solution irradiated in He-environment is shown in Fig.5.
Doses (kGy)

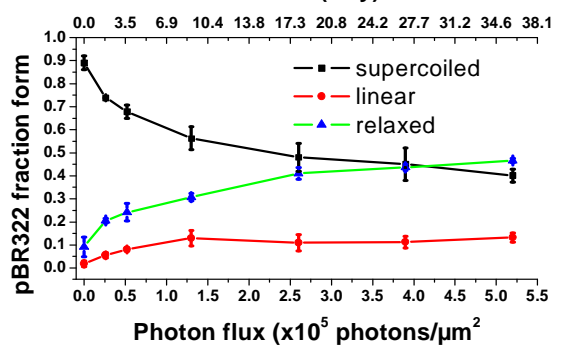

Figure 5.Fraction of yields of different forms of pBR22 plasmid DNA irradiated in He-environment as a function of photon flux. The dashes lines are to guide an eye.

Vertical error bars indicate the standard error of the mean. Horizontal errors are not included on the graph but correspond to about $10 \%$ random uncertainty on thephoton flux measurements on the photodiode and fluctuation in the density of the gases.

The results show that there was growing amount of SSB DNA with a corresponding decrease in the supercoiled form with the increasing dose of SXR radiation. Within experimental error, the fraction of the linear forms only increased at relatively few fluences of the soft X-rays (up to about $1.5 \times 10^{5}$ photons $/ \mu \mathrm{m}^{2}$ ) and then seems not to be monotonous in the helium condition irradiation at relatively pulses (i.e. higher photon fluence). The rapid increase in the linear form at relatively low photon fluence may be due to indirect damage from water radicals when the sample was much hydrated. At relatively longer exposure time, to achieve higher number of photons, the fraction of the linear form did not change much. Thus, the samples get dehydrated and may be the cause of the insignificant growth in the formation of the linear. It is therefore suspected that there might be the presence of free radicals formed by the irradiated plasmid in solution by the "water window" soft X-ray. This point can be further investigated by introducing radical scavengers to ascertain the presence of the free radicals created to induce further damage to the plasmid DNA. It will be a thing of concern in our forth coming articles.

The induction ratio of SSB to DSB in the helium irradiation condition was $\sim 5$, a value slightly lower than was measured when the samples were irradiated in vacuum. This may be explained as a result of the low penetration of this type of radiation through the relatively thick hydrated sample solution. This means that a very thin solution or a fraction of the solution (and hence the DNA) absorbed the radiation. Double strand breaks are considered the most critical DNA lesions induced by ionizing radiation. The dependence of the amount of linear and relaxed forms of the plasmid DNA on amount of photons is shown for the soft X-rays. Thus, the compact laser plasma water window $\mathrm{X}$-ray source induce exclusively radiation damage which provide an evidence for the ability of the water window X-ray radiation to induce both SSB and 
D. Adjei et al., Design and Characterization of a Desk-Top Laser ..., Rad. Applic., 2016, 1, 2, 88-94

DSB. The demonstration of the source to induce DSBs and SSBs in irradiated plasmid DNA shows the potential of the source for radiobiological experiments. Quantitative analysis of the radiation damage and its systematic investigation in DNA, biomembranes and other organelles, and living cells represent a subject of forthcoming articles.

\section{CONCLUSION}

A compact desktop laser plasma soft X-ray source based on a double-stream gas puff target operating in the "water window" spectral region has been presented. The source delivers approximately $6.83 \times 10^{13}$ photons $/ 4 \pi$ in the wavelength range of about 2.5-4 $\mathrm{nm}$. The low penetration depth of this radiation in biological samples and pulsed character of the source lead to high local dose loads and dose rates, respectively. The design of the source allows samples to be irradiated both in vacuum and in Heenvironment. Doses in a single pulse of soft X-rays of about $300 \mathrm{~Gy}$ for irradiation in vacuum and about 20 Gy for the He-environment irradiation were measured. Initial irradiation experiments carried out with plasmid DNA demonstrate that the source can be used in systematic studies of soft X-ray radiation damage to biomacromolecular samples and other biological specimen.

Acknowledgement: The authors acknowledge the financial support from the $\mathrm{EU} \mathrm{FP} 7$ Erasmus Mundus Joint Doctorate Programme EXTATIC under framework partnership agreement FPA2012-0033 and the support from Ghana Atomic Energy Commission. Appreciated is also the support from the 7th Framework Programme's Laserlab Europe project (No. 284464). PW greatly acknowledges the support by the National Centre for Science (Grant no. DEC2011/o3/D/ST2/oo296) and the National Centre for Research and Development, LIDER programme (Grant no. LIDER/oo4/41O/L-4/12/NCBR/2013). We also acknowledge Dr. B. Jankiewicz, Dr. P. Nyga and Mrs M. Gajda-Raczka for allowing us to use their chemistry laboratory for biological sample preparations.

\section{REFERENCES}

1. D.T. Goodhead and J. Thacker, "Inactivation and mutation of cultured mammalian cells by aluminium characteristic ultrasoft X-rays. I. Properties of aluminium X-rays and preliminary experiments with Chinese hamster cells," Int. J. Radiat. Biol., Vol. 31, pp.541-559, 1977.

2. D.T. Goodhead, J. Thacker, and R. Cox, "Effectiveness of $0.3 \mathrm{keV}$ carbon ultrasoft X-rays for inactivation and mutation of cultured mammalian cells," Int. J. Radiat. Biol., vol. 36, pp. 101-114, 1979.

3. D.J. Brenner, R. P. Bird, M. Zaider, P. Goldhagen, P. J. Kliauga, and H. H. Rossi, "Inactivation of synchronized mammalian cells with low-energy X-
rays-Results and significance," Radiat. Res., vol. 110, pp. 413-427, 1987.

4. K.M. Prise, M. Folkard, S. Davies, and B. D. Michael, "Measurements of DNA damage and cell killing in Chinese hamster V79-4 cells irradiated with aluminum characteristic ultrasoft X- rays," Radiat. Res., vol. 117, pp. 489-499, 1989.

5. D.T. Goodhead, "Soft X-ray radiobiology and synchrotron radiation," in Synchrotron Radiation in the Biosciences, Eds. Chance, B. et al., Clarendon Press, Oxford, 1994, pp. 683-705.

6. D. Frankenberg, H. Kuehn, M. Frankenberg-Schwager, W. Lenhard, and S. Beckonert, "0.3 keV carbon K ultrasoft X-rays are four times more effective than $\gamma^{-}$ rays when inducing oncogenic cell transformation at low doses," Int. J. Radiat. Biol., vol. 68, pp. 593-601, 1995.

7. C.S. Griffin,D. L. Stevens, and J. R K. Savage, "Ultrasoft $1.5 \mathrm{keV}$ aluminum $\mathrm{K} \mathrm{X}$ rays are efficient producers of complex chromosome exchange aberrations as revealed by fluorescence in situ hybridization," Radiat. Res., vol. 146, pp. 144-150, 1996.

8. B.E. Nelms, et al., "A comparison of cytotoxicity after whole- or partial-cell irradiation with synchrotronproduced ultrasoft X rays," Radiat. Res., vol. 150, pp.521-527, 1998.

9. M.A. Herve du Penhoat et al., "Lethal effect of carbon K-shell photoionizations in Chinese hamster V79 cell nuclei: Experimental method and theoretical analysis," Radiat. Res., vol. 151, pp. 649-658, 1999.

10. M.N. Gould et al., "Radiobiological studies using synchrotron-produced ultrasoft X-rays,"J. Radiat. Res., vol. 40:Suppl., pp. 66-73, 1999.

11. C. M. de Lara, M. A. Hill, T. J. Jenner, D. Papworth, and P. O'Neill, "Dependence of the yield of DNA double-strand breaks in Chinese hamster V79-4 cells on the photon energy of ultrasoft $\mathrm{X}$ rays," Radiat. Res., vol. 155, pp. 440-448, 2001.

12. G. Schettinoet al., "Low-dose studies of bystander cell killing with targeted soft X rays," Radiat. Res., vol. 160, pp. 505-511, 2003.

13. R.B Hawkins, "Mammalian Cell Killing by Ultrasoft X Rays and High-Energy Radiation: An Extension of the MK Model,” Radiat. Res., vol. 166, pp. 431-442, 2006.

14. L. Chen, S. P. Jiang, L. B. Wan, X. D. Ma and M. F. Li, "Radiobiological investigations of soft X-rays near carbon, nitrogen, oxygen K-shell edges on Aspergillusoryzae spores," Radiat. Prot. Dos., vol. 128(1), pp. 68-71, 2008.

15. C. Oven et al., "An ultrasoft X-ray multi-microbeam irradiation system for studies of DNA damage responses by fixed- and live-cell fluorescence microscopy," Eur. Biophys. J., vol.38, pp. 721-728, 2009.

16. T. Friedrich, M. Durante, and M. Scholz, "Modeling cell survival after irradiation with ultrasoft $\mathrm{X}$ rays using the giant loop binary lesion model," Rad. Res., vol. 181, pp. 485-494, 2014.

17. E. Alizadehet a., "Thymidine decomposition induced by low-energy electrons and soft X rays under N2 and O2 atmospheres," Rad. Res., vol. 181, pp. 629-640, 2014.

18. I. C. E. Turcu et al., "Optimisation of an excimer laserplasma soft X-ray source for applications in biophysics and medical physics," Phys. Med., vol. 10(3), pp. 9399, 1994.

19. D. Batani et al., "Biosystem response to soft-X-rays irradiation: non-monotonic effects in the relevant biological parameters of yeast cells," Il Nuovo Cim. D, vol. 18(5), pp. 657-662, 1996. 
D. Adjei et al., Design and Characterization of a Desk-Top Laser ..., Rad. Applic., 2016, 1, 2, 88-94

20. M. Nishikino et al., "Note: Application of laser produced plasma $\mathrm{K}$ alpha $\mathrm{x}$-ray probe in radiation biology," The Rev. Sci. Instrum., vol. 81(2), 026107, 2010.

21. H. Fiedorowicz, A. Bartnik, R. Jarocki, R. Rakowski, M. Szczurek, "Enhanced X-ray emission in the $1-\mathrm{keV}$ range from a laser-irradiated gas puff target produced using the double-nozzle setup," Appl. Phys. B, vol. 70, pp. 305-308, 2000.

22. P. W. Wachulak et al., "Water window" compact, tabletop laser plasma soft X-ray sources based on a gas puff target," Nucl. Instr. \& Meth. Phys. Res. B, vol. 268, pp. 1692-1700, 2010

23. M. Davídková et al., "A high-power laser-driven source of sub-nanosecond soft X-ray pulses for single-shot radiobiology experiments," Rad. Res., vol. 168, pp. 382-387, 2007.

24. Optical Constants.

Retrieved from: http://henke.lbl.gov/optical constants/; Retrieved on: Aug. 19, 2016.
25. D. Adjei et al., "Development of compact laserproduced plasma soft x-ray source for radiobiology experiments", submitted for publication.

26. A. Yokoya, R. Watanabe, and T. Hara, "Single- and double-strand breaks in solid pBR322 DNA induced by ultrasoft X-rays at photon energies of 388,435 and 573 eV," Rad. Res., vol. 40(2), pp. 145-158, 1999.

27. K. Hempel and E. Mildenberger , "Determination of Gvalues for single and double strand break induction in plasmid DNA using agarose gel electrophoresis and curve-fitting procedure," Int. J. Radiat. Biol., vol. 52(1), pp. 125-138, 1987.

28. S. J. McMahon and F. J. Currel, "A robust curve-fitting procedure for the analysis of plasmid DNA strand breaks data from gel electrophoresis," Radiat. Res., vol. 175, pp. 797-805, 2011.

29. A. Eschenbrenner, M. A. Herve Du Penhoat, A. Boissiere, G. Eot-Houllier, F. Abel, M.-F. Politis, A. Touati, E. Sage, and A. Chetioui, "Strand breaks induced in plasmid DNA by ultrasoft X-rays: influence of hydration and packing," Int. J. Radiat. Biol., vol. 83(10), pp. 687-697, 2007. 